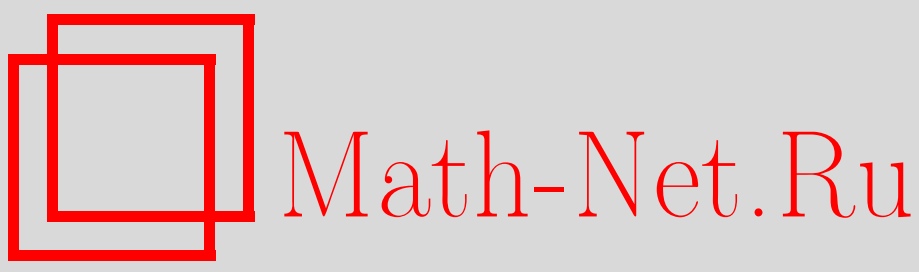

А. Ю. Хренников, Законы больших чисел в неархимедовой теории вероятностей, Изв. РАН. Сер. матем., 2000, том 64, выпуск 1,211-223

DOI: https://doi.org/10.4213/im280

Использование Общероссийского математического портала Math-Net.Ru подразумевает, что вы прочитали и согласны с пользовательским соглашением

http://www . mathnet.ru/rus/agreement

Параметры загрузки:

IP: 54.198 .67 .100

26 апреля 2023 г., 16:02:03 
УДК 519

А. Ю. Хренников

\section{Законы больших чисел в неархимедовой теории вероятностей}

\footnotetext{
Рассматриваются неколмогоровские вероятностные модели, а именно модели с $p$-адическими вероятностями. Получен аналог теоремы Бернулли для симметричной схемы.

Библиографйл: 9 наименований.
}

Новые физические исследования стимулируют развитие новых математических формализмов. В частности, исследования в неархимедовой математической физике (см. [1]) стимулировали построение ряда новых разделов неархимедовой математики. В работах автора [2]-[5] была развита модель теории вероятностей, в которой вероятности могут принадлежать полям $p$-адических чисел $Q_{p}$. В этой статье доказывается предельная теорема для сумм одинаково распределенных независимых случайных величин (СВ), которую (несмотря на существенные отличия) можно рассматривать как аналог обычной теоремы Бернулли (мы ограничиваемся симметричным случаем).

\section{$\S 1$. Вероятностное пространство Бернулли}

Пусть $\Omega_{B}$ - стандартное вероятностное пространство Бернулли, состоящее из последовательностей $\omega=\left(\omega_{j}\right)_{j=1}^{\infty}, \omega_{j}=0,1$. Пусть $I=\bigcup_{n} I_{n}$, где $I_{n}$ - множество всех векторов длины $n$ с координатами 0,1 . Пусть $i \in I_{n}$ и $B_{i}=\{\omega \in$ $\left.\Omega: \omega_{1}=i_{1}, \ldots, \omega_{n}=i_{n}\right\}$ - цилиндрическое множество с основанием $i$. Обозначим символом $F$ алгебру, порожденную цилиндрическими множествами. Стандартная мера Бернулли $\mu$ определяется на цилиндрических множествах равенством $\mu\left(B_{i}\right)=1 / 2^{n}, i \in I_{n}$, и продолжается по аддитивности на алгебру $F$. Эта мера $\sigma$-адлитивна и может быть продолжена до стандартной бернуллиевской вероятности $P_{\infty}$ на $\sigma$-алгебре $F_{\infty}$, порожденной $F .\left(\Omega_{B}, F_{\infty}, P_{\infty}\right)$ - вероятностное пространство в смысле аксиоматики Колмогорова.

ЗАмЕчАнИЕ 1.1. Символ $Q_{\infty}$ часто используется в теории чисел для обозначения поля вещественных чисел. Нам удобно использовать индекс $\infty$ при обозначении вещественных объектов.

Как обычно, положим $\xi_{n}(\omega)=\omega_{n}$. Это - независимые СВ,

$$
P_{\infty}\left(\xi_{n}(\omega)=\alpha\right)=\mu\left(\xi_{n}(\omega)=\alpha\right)=\frac{1}{2}, \quad \alpha=0,1 .
$$

Согласно теореме Бернулли для $T_{n}=S_{n} / n, S_{n}=\xi_{1}+\cdots+\xi_{n}$,

$$
T_{n}(\omega) \rightarrow \xi_{\lim }(\omega),
$$

(C) А.Ю. Хренников, 2000 
где $\xi_{\lim }(\omega)=1 / 2$ п.в. В частности, имеет место предельньй переход для распределений СВ $T_{n}(\omega)$ :

$$
P_{\infty, T_{n}} \rightarrow P_{\infty, \xi_{\text {lim }}},
$$

где предельное распределение равно $\delta_{1 / 2}$ (всюду символом $\delta_{a}$ обозначается $\delta$-мера Дирака, сосредоточенная в точке $a$ ), который означает слабую сходимость распределений:

$$
\mathbf{M} f\left(T_{n}(\omega)\right)=\int f(x) P_{\infty, T_{n}}(d x) \rightarrow f\left(\frac{1}{2}\right)
$$

для любой непрерывной ограниченной функции $f(x)$.

Далее,

$$
\mathbf{M} f\left(T_{n}(\omega)\right)=\sum_{k=0}^{n} f\left(\frac{k}{n}\right) \sum_{i_{1}+\cdots+i_{n}=k} \mu\left(B_{i}\right) .
$$

Следовательно,

$$
\mathbf{M} f\left(T_{n}(\omega)\right)=\frac{1}{2^{n}} \sum_{k=0}^{n} f\left(\frac{k}{n}\right) C_{n}^{k}
$$

где $C_{n}^{k}$ - биномиальные коэффициенты.

ЗАмечАниЕ 1.2. Эти выкладки для математического ожидания основаны только на цилиндрической мере $\mu$.

Таким образом, (1.3) может быть переписано как

$$
\lim _{n \rightarrow \infty} \frac{1}{2^{n}} \sum_{k=0}^{n} f\left(\frac{k}{n}\right) C_{n}^{k}=f\left(\frac{1}{2}\right) .
$$

Но нас интересует другое продолжение $\mu$. Так как $\mu$ принимает значения в $Q$, ее можно также рассматривать как $p$-адическизначную меру.

Всюду далее символом $Q_{p}$ обозначается поле $p$-адических чисел, $|\cdot|{ }_{p}$ - абсолютное значение на $Q_{p}, U_{r}(a)=\left\{x:|x-a|_{p} \leqslant r\right\}$ - шар радиуса $r>0$ с центром в точке $a, U_{r} \equiv U_{r}(0)$. Как обычно, $Z_{p}$ - кольцо цельх $p$-адических чисел, $Z_{p}=U_{1}$.

Далее дается краткое введение в теорию интегрирования по $Q_{p}$-значным мерам [6], [7]. Пусть $\Omega$ - компактное ультраметрическое пространство, т.е. имеет место усиленное неравенство треугольника:

$$
\rho(x, y) \leqslant \max [\rho(x, z), \rho(z, y)] .
$$

Шары $W_{r}(a)=\{x \in \Omega: \rho(x, a) \leqslant r\}$ являются одновременно открытыми и замкнутыми. Например, $p$-адическая метрика $\rho_{p}(x, y)=|x-y|_{p}$ является ультраметрикой, каждый шар $U_{r}(a)$ - компактное ультраметрическое пространство.

Обозначим символом $F(\Omega)$ алгебру открыто-замкнутых подмножеств $\Omega$. Мера $\nu$ на $\Omega$ - это конечно-аддитивная функция, $\nu: F(\Omega) \rightarrow Q_{p}$. Мера называется ограниченной, если

$$
\operatorname{var}(\nu)=\sup \left\{|\nu(A)|_{p}: A \in F(\Omega)\right\}<\infty .
$$

Обозначим символом $C\left(\Omega, Q_{p}\right)$ пространство непрерывных функций $f: \Omega \rightarrow Q_{p}$. Это $Q_{p}$-линейное банахово пространство с нормой $\|f\|=\max _{x \in \Omega}|f(x)|_{p}$. Имеет 
место взаимно однозначное соответствие между ограниченными мерами и ограниченными $Q_{p}$-линейными функционалами на пространстве $C\left(\Omega, Q_{p}\right)$ и $\|\nu\|=\operatorname{var}(\nu)$. Интеграл от непрерывной функции $f(x)$ по ограниченной мере $\nu$ определяется как предел сумм Римана:

$$
\int_{\Omega} f(x) \nu(d x)=\lim _{\alpha} \sum_{i=1}^{n} f\left(a_{i}\right) \nu\left(A_{i}\right)
$$

где $\alpha=\left(A_{i}\right)$ - покрытие $\Omega, A_{i} \in F(\Omega), A_{i} \cap A_{j}=\varnothing, a_{i} \in A_{i}$.

Ограниченная нормализованная мера $\nu: F(\Omega) \rightarrow Q_{p}, \nu(\Omega)=1$, назьвается $p$-адической вероятностью, где $\Omega$ - пространство элементарных исходов, $F(\Omega)$ алгебра событий, $(\Omega, F(\Omega), \nu)$ - $p$-адическая вероятностная модель. Случайные величины - это непрерывные функции $\xi: \Omega \rightarrow Q_{p}$. Математическое ожидание СВ определяется как интеграл по вероятности. Определение независимости СВ такое же, как и в аксиоматике Колмогорова. В частности, $\mathbf{M} \zeta \eta=\mathbf{M} \zeta \mathbf{M} \eta$ для независимых СВ. Пусть $\xi(\Omega) \subset U_{r}(a)$, вероятностное распределение СВ $\xi$ на шаре $U_{r}(a)$ определяется, как и в обычной теории вероятностей:

$$
\nu_{\xi}(D)=\nu\left(\xi^{-1}(D)\right), \quad D \in F\left(U_{r}(a)\right)
$$

Это - вероятность на $U_{r}(a) ;\left(U_{r}(a), F\left(U_{r}(a)\right), \nu_{\xi}\right)$ - вероятностная модель.

Теперь вернемся к мере Бернулли. Пространство Бернулли $\Omega_{B}$ изоморфно кольцу целых 2 -адических чисел $Z_{2}$, цилиндрические множества $B_{i}$ - это шары $U_{2^{-n}}(a)$, где $a$-произвольная точка $Z_{2}$ со свойством $a_{0}=i_{1}, \ldots, a_{n-1}=i_{n}$. Алгебра $F$ совпадает с алгеброй открыто-замкнутых множеств $F(\Omega)$, и мера Бернулли $\mu$ на $F$ может быть рассмотрена как $Q_{p}$-значная мера. Эта мера ограничена, если $p \neq 2$.

Таким образом, для $p=3,5, \ldots, 127, \ldots$ имеем $p$-адические вероятностные модели $\left(Z_{2}, F\left(Z_{2}\right), P_{p}\right)$, где $P_{p}=\mu-Q_{p}$-значные бернуллиевские вероятности.

Интересно, что вероятности $P_{\infty}$ и $P_{p}$ совпадают для всех событий $B_{i}$, зависящих лишш от конечного числа экспериментов. Таким образом, невозможно различить эти распределения на эксперименте. Но эти вероятности имеют различные асимптотические свойства.

Бернуллиевские CB $\xi_{n}$ - непрерывные функции на $Z_{2}$. Более того, как и в обычной теории, $P_{p}\left(\xi_{n}=\alpha\right)=\mu\left(\xi_{n}=\alpha\right)=1 / 2, \alpha=0,1$. Возможно изучение асимптотики сумм $S_{n}(\omega)$ или нормированных сумм $T_{n}(\omega)$. Мы будем изучать асимптотику подпоследовательностей $\left\{S_{n_{k}}(\omega)\right\}$; эта асимптотика существенно зависит от асимптотики подпоследовательности $\left\{n_{k}\right\}$ в $Q_{p}$. Слабая сходимость распределений определяется, как в обычной теории вероятностей.

Метод характеристических функций - это один из наиболее мощных методов доказательства предельных теорем. Однако в $p$-адическом случае отсутствует теорема о связи между сходимостью характеристических функций и распределений. Таким образом, нельзя применить технику характеристических функций непосредственно. Но такая теорема имеет место для сходимости обобщенных функций на неархимедовых пространствах [5], [8]. Поэтому сначала рассмотрим предельньй переход (1.2) для обобщенных функций и найдем предельные законы распределения, которые оказываются вероятностными мерами в хороших случаях. В этих случаях 
доказывается слабая сходимость вероятностных распределений. В рамках теории обобщенных функций удается получить более широкий класс предельных теорем, чем в рамках теории вероятностных мер. В частности, имеет место предельная теорема для обобщенной вероятности $P_{2}$, которая не является ограниченной мерой. Но в этом случае сходимость имеет место для более узкого класса функций.

\section{§2. Метод характеристических функций}

Мы используем теорию аналитических обобщенных функций на неархимедовых пространствах [5], [8]. Более удобно работать на пространстве комплексных $p$-адических чисел $C_{p}$. Здесь $C_{p}$ - это пополнение алгебраического замыкания $Q_{p}^{a}$ поля $p$-адических чисел $Q_{p}$ (см., например, [6]); $|\cdot|_{p}$ - продолжение $p$-адического абсолютного значения на $C_{p},\left|C_{p}\right|=\left\{r \in \mathbb{R}_{+}: r=|z|_{p}, z \in C_{p}\right\}$. Положим $U_{r}=\left\{z \in C_{p}:|z|_{p} \leqslant r\right\}$. Это шары в $C_{p}$ с центром в нуле.

Функция $f: U_{r} \rightarrow C_{p}, r \in\left|C_{p}\right|$, называется аналитической, если ряд

$$
f(x)=\sum_{n=0}^{\infty} f_{n} x^{n}, \quad f_{n} \in C_{p},
$$

сходится равномерно на $U_{r}$. Топология в пространстве $A_{r} \equiv A\left(U_{r}\right)$ функций, аналитических на шаре $U_{r}$, определяется неархимедовой нормой

$$
\|f\|_{r}=\max _{x \in U_{r}}|f(x)|_{p}
$$

Это - неархимедово банахово пространство. Функция $f: C_{p} \rightarrow C_{p}$ называется иелой, если она аналитична на любом шаре $U_{r}$. Топология в пространстве целых функций $A \equiv A\left(C_{p}\right)$ определяется системой норм $\left\{\|\cdot\|_{r}\right\}$. Последовательность целых функций $\left\{f_{n}\right\}$ сходится в $A$ тогда и только тогда, когда она сходится равномерно на любом шаре $U_{r}$. Это - неархимедово пространство Фреше с топологией проективного предела последовательности банаховых пространств:

$$
A=\lim _{r \rightarrow \infty} \operatorname{proj} A_{r}
$$

Функция $f$ называется аналитической в нуле, если сушествует такое $r$, что $f \in A_{r}$. Пространство $A_{0} \equiv A_{0}\left(C_{p}\right)$ функций, аналитических в нуле, наделяется топологией индуктивного предела, $A_{0}=\lim _{r \rightarrow 0}$ ind $A_{r}$.

Мы выбираем пространства аналитических функций $A$ и $A_{0}$ в качестве пространств основных функций, сопряженные пространства $A^{\prime}$ и $A_{0}^{\prime}$ - пространства обобщенных функций (аналитических распределений).

В дальнейшем будет использоваться

ПРЕДЛОЖЕНИЕ 2.1. Пусть $\nu$ - ограниченная мера на шаре $U_{r}$ в $Q_{p}$. Тогда функционал

$$
f \mapsto \nu(f)=\int_{U_{r}} f(x) \nu(d x), \quad f \in A,
$$

непрерывен на пространстве $A$. 
Таким образом, любой ограниченной мере соответствует распределение $\nu \in A^{\prime}$. Различным мерам соответствуют различные распределения.

Пусть $\left\{\gamma_{n}\right\}$ - последовательность распределений в пространстве $A^{\prime}$. Если эта последовательность сходится к распределению $\gamma$ в слабой топологии пространства $A^{\prime}$, то $\left\{\gamma_{n}\right\}$ назьвается $A$-слабо сходящейся $\kappa \gamma$. Это означает, что $\left(\gamma_{n}, f\right) \rightarrow$ $(\gamma, f)$ для любой целой аналитической функции $f$.

В силу предложения 2.1 можно говорить об $A$-слабой сходимости для вероятностных распределений.

ОпРЕДЕЛЕниЕ 2.1. Преобразование Лапласа распределения $g \in A_{0}^{\prime}$ определяется равенством $L(g)(y)=(g, \exp \{y x\})$.

Теорема 2.1 [8]. Преобразование Лапласа $L: A_{0}^{\prime} \rightarrow A$ является изоморфизмом.

Таким образом, имеется неархимедово исчисление Лапласа

$$
A_{0}^{\prime} \stackrel{L}{\longrightarrow} A, \quad A_{0} \stackrel{L^{\prime}}{\stackrel{2}{\longrightarrow}} A^{\prime}
$$

Преобразование Лапласа в неархимедовом случае имеет все свойства обычного преобразования Лапласа.

Если распределение $\gamma \in A^{\prime}$ является образом ограниченной меры на $U_{r}$, то

$$
L^{\prime}(\gamma)(y)=\int_{U_{r}} e^{x y} \gamma(d x)
$$

для достаточно малых $y \in C_{p}$.

Если $p \neq 2$, то вероятностные распределения $P_{p, T_{n_{k}}}$ случайных величин $T_{n_{k}}(\omega)$ - ограниченные меры на соответствуюших шарах, они определяют распределения, принадлежашие пространству $A^{\prime}$. Таким образом,

$$
\varphi_{n_{k}}(z)=\mathbf{M} \exp \left\{z T_{n_{k}}(\omega)\right\}=\int_{U_{r}} \exp \{z x\} P_{p, T_{n_{k}}}(d x)=L^{\prime}\left(P_{p, T_{n_{k}}}\right)(z) .
$$

Воспользовавшись независимостью СВ, имеем

$$
\varphi_{n_{k}}(z)=\prod_{j=1}^{n_{k}} \mathbf{M} e^{z \xi_{j}(\omega) / n_{k}}=\left[\left(1+e^{z / n_{k}}\right) / 2\right]^{n_{k}} .
$$

Если $p=2$, то мера Бернулли $\mu$ неограничена, но она может быть реализована как распределение $P_{2} \in A^{\prime}$ (см. [5], [8]). В этом случае также возможно ввести распределения $P_{2, T_{n_{k}}} \in A^{\prime}$. Формула (2.1) по-прежнему верна. Чтобы доказать ее, нужно лишь использовать то, что преобразование Лапласа свертки равно произведению преобразований Лапласа.

Положим $\varphi(z ; a)=\left[\left(1+e^{z / a}\right) / 2\right]^{a}$ для $a \in Z_{p}, a \neq 0$. Эта функция определена для достаточно малых $z$. Так как $L^{\prime}: A^{\prime} \rightarrow A_{0}$ - изоморфизм, то существует распределение $\varkappa_{a} \in A^{\prime}$ такое, что $L^{\prime}\left(\varkappa_{a}\right)(z)=\varphi(z ; a)$.

Положим $r(p)=1 / p$, если $p \neq 2$, и $r(2)=1 / 4$. 
ЛЕмма 2.1. Пусть $a \neq 0$ принадлежит $Z_{p} u\left\{n_{k}\right\}$ - последовательность натуральных чисел, $n_{k} \rightarrow$ в в $Q_{p}$. Тогда функциональная последовательность $\left\{\varphi_{n_{k}}(z)\right\}$ сходится $\kappa\{\varphi(z ; a)\}$ в пространстве $A_{0}\left(C_{p}\right)$.

ДокАЗАТЕЛЬСТво. Можно записать $n_{k}=m_{k}+a$, где $m_{k} \rightarrow 0$. Здесь $\left|n_{k}\right|_{p}=$ $|a|_{p}$ для достаточно больших $k$. Далее,

$$
\varphi_{n_{k}}(z)=\left[\frac{1+e^{z / n_{k}}}{2}\right]^{a}\left[\frac{1+e^{z / n_{k}}}{2}\right]^{m_{k}}=\left(1+\Delta_{n_{k}}\right)^{a}\left(1+\Delta_{n_{k}}\right)^{m_{k}}
$$

Далее имеем

$$
\begin{aligned}
\mid(1 & \left.+\Delta_{n_{k}}\right)^{a}-\left.\left[1+\frac{e^{z / a}-1}{2}\right]^{a}\right|_{p} \\
& =\left|\exp \left\{a \ln \left(1+\Delta_{n_{k}}\right)\right\}-\exp \left\{a \ln \left(1+\frac{e^{z / a}-1}{2}\right)\right\}\right|_{p} \\
& =|a|_{p}\left|\ln \left(1+\Delta_{n_{k}}\right)-\ln \left(1+\frac{e^{z / a}-1}{2}\right)\right|_{p}=\left|\frac{a}{2}\right|_{p}\left|e^{z / n_{k}}-e^{z / a}\right|_{p} \\
& =\frac{\left|m_{k}\right|_{p}|z|_{p}}{|2 a|_{p}} \rightarrow 0, \quad k \rightarrow \infty
\end{aligned}
$$

где предельный переход имеет место равномерно на шаре $U_{r}, r=r(p)|a|_{p}$. Здесь мы использовали изометрические свойства экспоненты $e^{z}$ и логарифма $\ln (1+z)$ на $U_{r}$ (см., например, [6]). Отметим, что все функции определены при $z \in U_{r}$.

Аналогично доказывается, что $\left|\left(1+\Delta_{n_{k}}\right)^{m_{k}}-1\right|_{p} \rightarrow 0$ на том же шаре.

Следуюшая теорема является простым следствием (см. [5], [8]) леммы 2.1.

Теорема 2.2 (теорема Бернулли для обобщенных функций). Пусть последовательность натуральных чисел $\left\{n_{k}\right\}$ стремится $\kappa a \in Z_{p}, a \neq 0$. Тогда последовательность распределений $\left\{P_{p, T_{n_{k}}}\right\}$ сходится $A$-слабо к распределению $\varkappa_{a}$.

ПримеР 2.1. Пусть $a=1$. Тогда $\varkappa_{1}=\left(\delta_{0}+\delta_{1}\right) / 2$. Таким образом, предельное распределение последовательности $\left\{T_{n_{k}}(\omega)\right\}$ совпадает с начальньм распределением $\mathrm{CB} \zeta_{n}(\omega)$. Если, например, выбрать $n_{k}=1+p^{k}$, то

$$
\frac{\xi_{1}(\omega)+\cdots+\xi_{n_{k}}(\omega)}{1+p^{k}} \rightarrow \xi_{\lim }(\omega)
$$

где $\xi_{\lim }(\omega)-\mathrm{CB}$ с распределением $\varkappa_{1}$. Когда вьчислялось среднее значение (1.4) по цилиндрической мере Бернулли $\mu$, использовались лишь рациональные числа. Следовательно, эта формула остается верной и в $p$-адическом случае. Итак,

$$
\frac{1}{2^{p^{k}+1}} \sum_{j=0}^{1+p^{k}} f\left(\frac{j}{1+p^{k}}\right) C_{1+p^{k}}^{j} \rightarrow \frac{1}{2}(f(0)+f(1))
$$

для любой целой аналитической функции $f$. 
Далее, пусть $p=3$. Тогда $n_{k}=4^{3^{k}} \rightarrow 1$ и

$$
\frac{\xi_{1}(\omega)+\cdots+\xi_{4^{3}}(\omega)}{4^{3^{k}}} \rightarrow \xi_{\lim }(\omega)
$$

Эта асимптотика означает, что

$$
\left(\frac{1}{2^{4^{3^{k}}}}\right) \sum_{j=0}^{4^{3^{k}}} f\left(\frac{j}{4^{3^{k}}}\right) C_{4^{3^{k}}}^{j} \rightarrow \frac{1}{2}(f(0)+f(1)) .
$$

Если $p=2$, то, например, $n_{k}=(2 m+1)^{2^{k}} \rightarrow 1$ для $m=1,2, \ldots$, следовательно,

$$
\frac{\xi_{1}(\omega)+\cdots+\xi_{(2 m+1)^{2^{k}}}(\omega)}{(2 m+1)^{2^{k}}} \rightarrow \xi_{\lim }(\omega) .
$$

ПримеР 2.2. Пусть $a=2$. Тогда предельное распределение имеет вид $\varkappa_{2}=$ $(1 / 4) \delta_{0}+(1 / 2) \delta_{1 / 2}+(1 / 4) \delta_{1}$. Более того, если $a=m$ - натуральное число, то предельное распределение имеет вид

$$
\varkappa_{m}=\frac{1}{2^{m}} \sum_{j=0}^{m} C_{m}^{j} \delta_{j / m} .
$$

Таким образом, если $n_{k}=m+p^{k}$, то

$$
\frac{1}{2^{m+p^{k}}} \sum_{j=0}^{m+p^{k}} C_{m+p^{k}}^{j} f\left(\frac{j}{m+p^{k}}\right) \rightarrow \frac{1}{2^{m}} \sum_{j=0}^{m} C_{m}^{j} f\left(\frac{j}{m}\right)
$$

для любой целой аналитической функции $f$.

\section{§ 3. Теорема Бернулли для вероятностных распределений}

Теперь мы хотим доказать аналог теоремы 2.2 для слабой сходимости вероятностных распределений. Следовательно, рассматривается случай $p \neq 2$. Сначала будет найдена асимптотика сумм $S_{n_{k}}(\omega)$, а затем нормированных сумм $T_{n_{k}}(\omega)$. Заметим, что $\left|S_{n}(\omega)\right|_{p} \leqslant 1$. Следовательно, все вероятностные распределения $P_{p, S_{n}}$ сосредоточены на $Z_{p}$. Удобно воспользоваться теорией Малера [9], [6] интегрирования по кольцу целых $p$-адических чисел $Z_{p}$.

Рассмотрим биномиальные функции

$$
C(x, k)=C_{x}^{k}=\frac{x(x-1) \ldots(x-k+1)}{k !} .
$$

Любая функция $f \in C\left(Z_{p}, Q_{p}\right)$ раскладывается в ряд (разложение Малера):

$$
f(x)=\sum_{k=0}^{\infty} a_{k} C(x, k) .
$$

Этот ряд сходится равномерно на $Z_{p}$, потому что $a_{k} \rightarrow 0, k \rightarrow \infty$, и $\|f\|=$ $\max _{k}\left|a_{k}\right|_{p}$. Если $\nu$ - ограниченная мера на $Z_{p}$, то

$$
\int_{Z_{p}} f(x) \nu(d x)=\sum_{k=0}^{\infty} a_{k} \int_{Z_{p}} C(x, k) \nu(d x) .
$$

Положим $\lambda_{m n}=\mathbf{M} C\left(S_{n}(\omega), m\right)=\int_{Z_{p}} C(x, k) P_{p, S_{n}}(d x)$.

ЛЕмма 3.1. Если $m \leqslant n$, то $\lambda_{m n}=C_{n}^{m} / 2^{m}$, если $m>n$, mo $\lambda_{m n}=0$. 
ДоКАЗАТЕЛЬСТВо. Воспользуемся формулой [6]

$$
e^{t x}=\sum_{m=0}^{\infty}\left(e^{t}-1\right)^{m} C(x, m) .
$$

Таким образом,

$$
\mathbf{M} \exp \left\{t S_{n}(\omega)\right\}=\sum_{m=0}^{\infty}\left(e^{t}-1\right)^{m} \lambda_{m n}
$$

Далее,

$$
\mathbf{M} \exp \left\{t S_{n}(\omega)\right\}=\left[\frac{1+e^{t}}{2}\right]^{n}=\sum_{m=0}^{n} C_{n}^{m} \frac{\left(e^{t}-1\right)^{m}}{2^{m}}
$$

Теперь покажем, что обобщенная функция $\varkappa_{a}$, определенная в предыдущем параграфе с помощью преобразования Лапласа, является ограниченной мерой на шаре $U_{1 /|a|_{p}}$. Рассмотрим сначала функцию $\psi(z ; a)=\left[\left(1+e^{z}\right) / 2\right]^{a}$. Этой функции также соответствует обобщенная функция, принадлежащая $A^{\prime}\left(C_{p}\right)$. Обозначим ее символом $\alpha_{a} ;$ здесь $a \in Z_{p}$ любое (в частности, $a=0$ ).

ЛЕмма 3.2. Имеют место равенства

$$
\left(\alpha_{a}, C(\cdot, m)\right)=\frac{C(a, m)}{2^{m}} .
$$

ЛЕмма 3.3. Обобщенной функиии $\alpha_{a}$ соответствует ограниченная мера на $Z_{p}$.

ДоказАТЕЛЬСТво. Если $a \in Z_{p}$, то $|C(a, m)|_{p} \leqslant 1$ (см. [6]). Остается воспользоваться леммой 3.2 .

Заметим, что

$$
\int_{Z_{p}} f(x) \alpha_{a}(d x)=\sum_{m=0}^{\infty} \frac{a_{m} C(a, m)}{2^{m}} .
$$

Рассмотрим функционал на пространстве $C\left(U_{1 /|a|_{p}}, Q_{p}\right), a \neq 0$,

$$
\left(\beta_{a}, g\right)=\int_{Z_{p}} g\left(\frac{x}{a}\right) \alpha_{a}(d x)
$$

Этот функционал ограничен, т.е. $\beta_{a}$ - ограниченная мера на шаре $U_{1 /|a|_{p}}$.

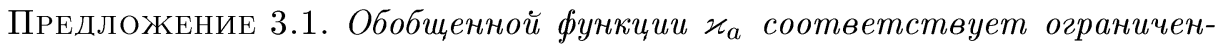
ная мера на шаре $U_{1 /|a|_{p}}$.

ДокАЗАТЕЛЬСтво. Вычисляя преобразования Лапласа, получаем

$$
L^{\prime}\left(\beta_{a}\right)(t)=\int_{Z_{p}} e^{t x / a} \alpha_{a}(d x)=\varphi(t ; a) .
$$

Итак, $\varkappa_{a}=\beta_{a}$. 
Tеорема 3.1 (теорема Бернулли для ненормированных сумм). Пусть последовательность натуральных чисел $\left\{n_{k}\right\}$ сходится $к$ челому $р$-адическому числу а. Тогда для любой $f \in C\left(Z_{p}, Q_{p}\right)$

$$
\mathbf{M} f\left(S_{n_{k}}(\omega)\right)=\int_{Z_{p}} f(x) P_{p, S_{n_{k}}}(d x) \rightarrow \int_{Z_{p}} f(x) \alpha_{a}(d x) .
$$

ДокАЗАТЕЛьство. Для среднего значения имеем

$$
\mathbf{M} f\left(S_{n_{k}}(\omega)\right)=\sum_{m=0}^{n_{k}} a_{m} \lambda_{m n_{k}} .
$$

Для достаточно большого $N$ получаем $\max _{N<m<\infty}\left|a_{m}\right|_{p}<\varepsilon$. Поэтому достаточно оценить

$$
\sum_{m=0}^{N} a_{m}\left(\lambda_{m n_{k}}-\frac{C(a, m)}{2^{m}}\right) .
$$

Но коэффициенты $a_{m}$ ограничены нормой $\|f\|$. Таким образом, достаточно показать, что $\lambda_{m n_{k}} \rightarrow C(a, m) / 2^{m}$ при фиксированном $m$. Но

$$
\lambda_{m n_{k}}=\frac{n_{k}\left(n_{k}-1\right) \ldots\left(n_{k}-m+1\right)}{m ! 2^{m}},
$$

и остается перейти к пределу при $n_{k} \rightarrow a$.

ЛЕмма 3.4. Пусть $a \neq 0$. Тогда для любой функции $f \in C\left(U_{1 /|a|_{p}}, Q_{p}\right)$

$$
\max _{\omega \in Z_{2}}\left|\frac{S_{n_{k}}(\omega)}{a}-T_{n_{k}}(\omega)\right|_{p} \rightarrow 0, \quad n_{k} \rightarrow a
$$

ТЕорема 3.2 (теорема Бернулли для нормированных сумм). Пусть последовательность натуральных чисел $\left\{n_{k}\right\}$ сходится к иелому р-адическому числу $a$, отличному от нуля. Тогда для любой функиии $f \in C\left(U_{1 /|a|_{p}}, Q_{p}\right)$

$$
\mathbf{M} f\left(T_{n_{k}}(\omega)\right)=\int_{U_{1 /|a| p}} f(x) P_{p, T_{n_{k}}}(d x) \rightarrow \int_{U_{1 /|a| p}} f(x) \varkappa_{a}(d x) .
$$

ДокАЗАТЕЛЬСтво. Заметим, во-первых, что для $f \in C\left(U_{1 /|a|_{p}}, Q_{p}\right)$

$$
\mathbf{M} f\left(\frac{S_{n_{k}}(\omega)}{a}\right) \rightarrow \int_{Z_{p}} f\left(\frac{x}{a}\right) \alpha_{a}(d x)=\int_{U_{1 /|a| p}} f(x) \varkappa_{a}(d x) .
$$

Далее,

$$
\left|\mathbf{M} f\left(\frac{S_{n_{k}}(\omega)}{a}\right)-\mathbf{M} f\left(T_{n_{k}}(\omega)\right)\right|_{p} \leqslant \operatorname{var} P_{p} \max _{\omega \in Z_{2}}\left|f\left(\frac{S_{n_{k}}(\omega)}{a}\right)-f\left(T_{n_{k}}(\omega)\right)\right|_{p} .
$$


Остается воспользоваться леммой 3.4 и равномерной непрерывностью функции $f(x)$.

Заметим, что если функция $f(x)$ принимает только рациональные значения в рациональных точках, то выражение для среднего $\mathbf{M} f\left(T_{n}(\omega)\right)$ одно и то же как в обычной теории вероятностей над полем вещественных чисел, так и в $p$-адических теориях (см. формулу (1.4)). Таким образом, теорема 3.2 показывает, что известные вероятностные средние по симметричному биномиальному распределению, кроме обычной вешественной асимптотики, имеют еще дополнительные $p$-адические асимптотики.

Например, пусть $f(x)=x^{l}$. Тогда в силу обычной теоремы Бернулли

$$
\sigma(n, l)=\frac{1}{2^{n}} \sum_{j=0}^{n} C_{n}^{j}\left(\frac{j}{n}\right)^{l} \rightarrow\left(\frac{1}{2}\right)^{l}, \quad n \rightarrow \infty .
$$

Теперь если $n_{k} \rightarrow 1$ в $Q_{p}$, то $\sigma\left(n_{k}, l\right) \rightarrow 1 / 2$ в $Q_{p}$. Если же $n_{k} \rightarrow 2$, то $\sigma\left(n_{k}, l\right) \rightarrow$ $1 / 2^{l+1}+1 / 4$.

Далее будем выбирать в качестве $f(x)$ индикаторы открыто-замкнутых множеств $A \in F\left(Z_{p}\right)$. Тогда для $n_{k} \rightarrow 1$ имеем

$$
P_{p}\left(\left\{\omega \in \Omega_{B}: T_{n_{k}}(\omega) \in A\right\}\right) \rightarrow \frac{\chi_{A}(0)+\chi_{A}(1)}{2} .
$$

Пусть $A=U_{\varepsilon}, \varepsilon<1$; тогда

$$
\begin{aligned}
P_{p}\left(\left|\frac{\xi_{1}(\omega)+\cdots+\xi_{n_{k}}(\omega)}{n_{k}}\right|_{p} \leqslant \varepsilon\right) & \rightarrow \frac{1}{2}, k \rightarrow \infty, \\
P_{p}\left(\left|\frac{\xi_{1}(\omega)+\cdots+\xi_{n_{k}}(\omega)}{n_{k}}-1\right|_{p} \leqslant \varepsilon\right) & \rightarrow \frac{1}{2}, \quad k \rightarrow \infty .
\end{aligned}
$$

Если $c \neq 0,1$, то для достаточно малого $\varepsilon$

$$
P_{p}\left(\left|\frac{\xi_{1}(\omega)+\cdots+\xi_{n_{k}}(\omega)}{n_{k}}-c\right|_{p} \leqslant \varepsilon\right) \rightarrow 0, \quad k \rightarrow \infty .
$$

Можно рассматривать эти равенства как $p$-адический аналог закона больших чисел.

Рассмотрим теперь индикаторы $p$-адических сфер $S_{r}=\left\{x \in Z_{p}:|x|_{p}=r\right\}$, $r=1 / p^{l}, l=0,1,2, \ldots$ :

$$
P_{p}\left(\left|\frac{\xi_{1}(\omega)+\cdots+\xi_{n_{k}}(\omega)}{n_{k}}\right|_{p}=1\right) \rightarrow \frac{1}{2}, \quad k \rightarrow \infty
$$

при $l=1,2, \ldots$

$$
P_{p}\left(\left|\frac{\xi_{1}(\omega)+\cdots+\xi_{n_{k}}(\omega)}{n_{k}}\right|_{p}=\frac{1}{p^{l}}\right) \rightarrow 0, \quad k \rightarrow \infty
$$




\section{§4. Комбинаторные следствия $p$-адической теоремы Бернулли}

Как известно, обычная теорема Бернулли имеет простой комбинаторный смысл. Посмотрим, как обстоят дела в $p$-адическом случае. Ограничимся подпоследовательностями $n_{k} \rightarrow 1$. Здесь

$$
A_{l, n}=\left\{\omega:\left|T_{n_{k}}(\omega)\right|_{p}=\frac{1}{p^{l}}\right\}=\left\{\omega:\left|S_{n_{k}}(\omega)\right|_{p}=\frac{1}{p^{l}}\right\}
$$

Каков смысл событий $A_{l, n}$ ? Событие $A_{l, n}$ состоит в том, что сумма единищ, выпавших в течение $n$ испытаний, делится ровно на $p^{l}$ (т.е. на $p^{l+1}$ уже не делится). Асимптотика (3.6) означает, что $p$-адическая вероятность события $A_{l, n_{k}}$, $l \neq 0$, стремится к нулю, $k \rightarrow \infty$. А это равносильно тому, что $P_{p}\left(A_{l, n_{k}}\right)$ делится на все большую степень $p$ с ростом $k$. Однако эту вероятность можно подсчитать непосредственно с помошью простых комбинаторных выкладок. Пусть $m(l, n)$ - количество $i \in I_{n}$, для которых $|i|=i_{1}+\cdots+i_{n}$ делится ровно на $p^{l}$. Несложно выписать выражение для $m(l, n)$ через сумму $C_{n}^{j}$. Таким образом, $P_{p}\left(A_{l, n}\right)=m(l, n) / 2^{n}$. Но $2^{n}$ не делится на $p$, значит, асимптотика (3.6) эквивалентна тому, что $m\left(l, n_{k}\right)$ делится на все большую степень $p$ с ростом $k$.

Далее, $P_{p}\left(A_{0, n}\right)=m(0, n) / 2^{n}$, и асимптотика (3.5) означает, что $m\left(0, n_{k}\right) / 2^{n_{k}} \rightarrow 1 / 2$ в $Q_{p}$. Например, $p=3$ и $n_{k}=1+3^{k}$. Тогда $2^{n_{k}} \rightarrow-2$. Итак, $m\left(0, n_{k}\right) \rightarrow-1$, т.е. $m\left(0, n_{k}\right)+1 \rightarrow 0$. Следовательно, $m\left(0, n_{k}\right)+1$ делится на все большую степень $p$ с ростом $k$.

Заметим, что асимптотику (3.6) несложно найти непосредственно из комбинаторного выражения для вероятности. Например, $m(1, n)$ равно сумме $C_{n}^{j p}$, где $j$ не делится на $p$, но

$$
\begin{aligned}
\left|m\left(1,1+p^{k}\right)\right|_{p} & \leqslant \max _{j}\left|C_{1+p^{k}}^{j p}\right|_{p} \\
& =\left|\frac{p^{k}}{p j} \cdot \frac{p^{k}-p}{p} \cdot \frac{p^{k}-2 p}{2 p} \cdots \frac{p^{k}-p(j-1)}{(j-1) p}\right|_{p}=\frac{1}{p^{k-1}} .
\end{aligned}
$$

Здесь мы получаем уточнение асимптотики (3.6). А именно, вероятность $P_{p}\left(A_{1,1+p^{k}}\right)$ уменьшается в $p$ раз после каждого шага. Этот результат несложно обобщить на любую последовательность $n_{k} \rightarrow 1$ в $Q_{p}$. Действительно, $n_{k}=$ $1+j_{k} p^{m_{k}}$, где $m_{k} \rightarrow \infty, k \rightarrow \infty, j_{k}$ - натуральные числа, которые не делятся на $p$. Тогда

$$
\left|C_{n_{k}}^{p j}\right|_{p}=\left|\frac{\left(p^{m_{k}} j_{k}\right)\left(p^{m_{k}} j_{k}-p\right) \ldots\left(p^{m_{k}} j_{k}-p(j-1)\right)}{p(2 p) \ldots(j-1) p \cdot(j p)}\right|_{p}=\frac{1}{p^{m_{k}-1}} .
$$

Здесь после каждого шага вероятность события $P_{p}\left(A_{1, n_{k}}\right)$ уменьшается в $p^{m_{k+1}-m_{k}}$ раз. Теперь рассмотрим событие $A_{l, n_{k}}, l \neq 0$. Здесь

$$
\left|C_{n_{k}}^{p^{l} j}\right|_{p}=\left|\frac{\left(p^{m_{k}} j_{k}\right) \ldots\left(p^{m_{k}} j_{k}-p^{l} j+p\right)}{p \ldots\left(p^{l} j-p\right) p^{l} j}\right|_{p}=\frac{1}{p^{m_{k}-l}} .
$$

В частности, если $n_{k}=1+p^{k}$, то имеем $1 / p^{k-l}$. 
С помошью прямых комбинаторных выкладок мы можем получить общую предельную теорему (не вытекающую из теоремы 3.2). Для этого нам понадобится две технические леммы.

Для любых $n, k \in \mathbb{N}$ обозначим через $(n, k)$ наибольший обший делитель этих чисел. Для любого $n \in \mathbb{N}$ обозначим через $M_{p}(n)$ вычет по модулю $p$ числа $n$ : $n=M_{p}(n) \bmod p$. Мы полагаем

$$
\theta_{p}(n)= \begin{cases}\left|n-M_{p}(n)\right|_{p}, & n \geqslant p \\ 1, & 1 \leqslant n \leqslant p-1\end{cases}
$$

ЛЕмма 4.1. Пусть $n, k \in \mathbb{N}, \quad k \leqslant n, u M_{p}(n) \geqslant M_{p}^{k}(k)$. Тогда

$$
\left|C_{n}^{k}\right|_{p}=\frac{\theta_{p}(n)}{\theta_{p}(k)}
$$

ДокАЗАТЕльСтво. Пусть $n=\alpha+i p^{N}, k=\beta+j p^{l}$, где $0 \leqslant \alpha, \beta \leqslant p-1$, $i, j, N, l \in \mathbb{N},(i, p)=(j, p)=1$. Мы имеем

$$
\left|C_{n}^{k}\right|_{p}=\left|\left(i p^{N}\right) \cdot \frac{\left(i p^{N}-p\right)}{p} \cdots \frac{\left(i p^{N}-j p^{l}+p\right)}{\left(j p^{l}-p\right)} \cdot \frac{1}{\left(j p^{l}\right)}\right|_{p}=\left|\frac{p^{N}}{p^{l}}\right|_{p}=p^{l-N}
$$

Для получения равенства (4.2) мы использовали следуюшие рассуждения: поскольку $n-k+1=i p^{N}-j p^{l}+(\alpha+1-\beta), 0<\alpha+1-\beta \leqslant p$, последний член в числителе биномиального коэффициента $C_{n}^{k}=\frac{n \cdots(n-k+1)}{1 \cdots k}$, которьй делится на $p$, равен $\left(i p^{N}-j p^{l}+p\right)$. Случаи $n=\alpha$ или $k=\beta, 0 \leqslant \alpha, \beta \leqslant p-1$, рассматриваются аналогично.

Лемма 4.2. Пусть $n, k \in \mathbb{N}, k \leqslant n, u M_{p}(n)+1 \leqslant M_{p}(k)$. Тогдa

$$
\left|C_{n}^{k}\right|_{p}=\theta_{p}(n)
$$

ДокАЗАТЕЛЬСТво. Пусть $n=\alpha+i p^{N}, k=\beta+j p^{l}$, где $(i, p)=(j, p)=1$, $0 \leqslant \alpha, \beta \leqslant p-1$. Имеем

$$
\left|C_{n}^{k}\right|_{p}=\left|\left(i p^{N}\right) \frac{\left(i p^{N}-p\right)}{p} \ldots \frac{\left(i p^{N}-j p^{l}\right)}{j p^{l}}\right|_{p}=\left|p^{N}\right|_{p}=p^{-N} .
$$

Для получения равенства (4.4) мы использовали следующие рассуждения: поскольку $n-k+1=\left(i p^{N}-j p^{l}\right)-(\beta-\alpha-1), 0 \leqslant \beta-\alpha-1<p$, последний член в числителе биномиального коэффициента $C_{n}^{k}=\frac{n \cdots(n-k+1)}{1 \cdots k}$, который делится на $p$, равен $\left(i p^{N}-j p^{l}\right)$. Случаи $n=\alpha$ или $k=\beta, 0 \leqslant \alpha, \beta \leqslant p-1$, рассматриваются аналогично. 
ТЕОрема 4.1. Для любого $l \in \mathbb{N}$ р-адическая вероятность

$$
P_{p}\left(\left\{\omega:\left|S_{n}(\omega)-M_{p}\left(S_{n}(\omega)\right)\right|_{p}=\frac{1}{p^{l}}, M_{p}\left(S_{n}(\omega)\right) \leqslant M_{p}(n)\right\}\right)
$$

стремится к нулю в $Q_{p}$, если $\left|n-M_{p}(n)\right|_{p} \rightarrow 0, \quad n \neq M_{p}(n)$.

ДокАЗАТЕльСтво. Используя лемму 3.1, получаем

$$
P_{p}\left(\left\{\omega:\left|S_{n}(\omega)-M_{p}\left(S_{n}(\omega)\right)\right|_{p}=\frac{1}{p^{l}}, M_{p}\left(S_{n}(\omega)\right) \leqslant M_{p}(n)\right\}\right) \leqslant p^{l}\left|n-M_{p}(n)\right|_{p} .
$$

ТЕОРема 4.2. р-адическая вероятность

$$
P_{p}\left(\left\{\omega: M_{p}\left(S_{n}(\omega)\right) \geqslant M_{p}(n)+1\right\}\right)
$$

стремится к нулю при $\left|n-M_{p}(n)\right|_{p} \rightarrow 0$.

Доказательство опирается на лемму 3.2 .

СлЕДСТвИЕ 4.1. p-адическая вероятность $P_{p}\left(\left\{\omega: M_{p}\left(S_{n}(\omega)\right) \geqslant 1\right\}\right)$ стремится к нулю при $|n|_{p} \rightarrow 0$.

СлЕДСТВИЕ 4.2. $р$-адическая вероятность $P_{p}\left(\left\{\omega: M_{p}\left(S_{n}(\omega)\right) \geqslant 2\right\}\right)$ стремится к нулю при $|n-1|_{p} \rightarrow 0$.

\section{Список литературы}

1. Владимиров В. С., Волович И. В., Зеленов Е. И. p-адический анализ и математическая физика. М.: Наука, 1994.

2. Хренников А. Ю. p-адическая вероятность и статистика // Докл. РАН. 1992. Т. 332. № 6. C. $1075-1079$.

3. Хренников А.Ю. Неархимедова вероятность: Сб. статей К 70-летию академика В. С. Владимирова // Тр. МИАН. 1994. Т. 203. С. 184-193.

4. Хренников А. Ю. $p$-адическая теория вероятностей и ее приложения. Принцип статистической стабилизации частот // ТМФ. 1993. Т. 97. № 3. С. 348-363.

5. Khrennikov A. Yu. p-adic valued distributions in mathematical physics. Dordrecht-Boston-London: Kluwer Academic Publ., 1994.

6. Schikhov W. Ultrametric Calculus. Cambridge: Cambridge Univ. Press, 1984.

7. Monna A. Analyse non-Archimedienne. Berlin: Springer, 1970.

8. Хренников А.Ю. Обобщенные функции и гауссовские континуальные интегралы по неархимедовым функциональным пространствам // Изв. РАН. Сер. матем. 1991. Т. 55. № 4. C. $780-814$.

9. Mahler K. p-adic numbers and their functions. Cambridge: Cambridge Univ. Press, 1980. 